\title{
Role of endoplasmic reticulum stress on cisplatin resistance in ovarian carcinoma
}

\author{
JING TIAN, RONG LIU and QUANXIN QU \\ Department of Gynaecology, Tianjin First Central Hospital, Tianjin 300192, P.R. China
}

Received June 1, 2015; Accepted October 14, 2016

DOI: $10.3892 / \mathrm{ol} .2017 .5580$

\begin{abstract}
Endoplasmic reticulum (ER) is an essential site of cellular homeostasis regulation. ER stress (ERS) may induce autophagy in tumor cells that escape from apoptosis. The present study examined the effects and mechanism of ERS on cisplatin (DDP) sensitivity in ovarian carcinoma. SKOV3 tumor cells treated with Saquinavir were subjected to western blot and reverse transcription-quantitative polymerase chain reaction analysis to determine protein and messenger RNA (mRNA) expression levels of mechanistic target of rapamycin (mTOR) and Beclin 1. MTT assay was used to analyze the influence of Saquinavir on DDP resistance in SKOV3 cells. Saquinavir induced glucose-regulated protein 78 expression, which is a marker of ERS. Following treatment with various doses of Saquinavir, the sensitivity of ovarian cancer cells to DDP decreased significantly. Protein and mRNA expression levels of mTOR and Beclin 1 in SKOV3 cells were increased when the cells were exposed to Saquinavir or DDP for $24 \mathrm{~h}$. Moreover, mTOR and Beclin 1 expression levels were highest in the Saquinavir + DDP group $(0.684 \pm 0.072$ and $0.6467 \pm 0.0468$, respectively). SKOV3 tumor cells were also exposed to the autophagy inhibitor, 3-methyladenine (3-MA), and different concentrations of Saquinavir. Analysis of half maximal inhibitory concentration $\left(\mathrm{IC}_{50}\right)$ values of DDP after this treatment demonstrated that $\mathrm{IC}_{50}$ values were significantly decreased compared with Saquinavir alone $(\mathrm{P}<0.001)$, suggesting that the sensitivity to DDP was improved in ovarian cancer cells after 3-MA exposure. These findings demonstrated that Saquinavir is able to induce ERS in SKOV3 cells effectively, and ER-induced stress may decrease the sensitivity of DDP in SKOV3 cells. Furthermore, ERS may regulate cell autophagy through the mTOR and Beclin 1 pathways, leading to a reduction in the sensitivity of DDP in SKOV3 cells. ERS in tumor cells and autophagy may be a potential target to
\end{abstract}

Correspondence to: Dr Quanxin Qu, Department of Gynaecology, Tianjin First Central Hospital, 24 Fukang Road, Nankai, Tianjin 300192, P.R. China

E-mail: qqxin95425@126.com

Key words: ovarian carcinoma, cisplatin, drug resistance, endoplasmic reticulum stress, autophagy improve the therapeutic effect of chemotherapy and reduce drug resistance in tumors.

\section{Introduction}

Ovarian cancer is one of the most dangerous female malignant tumors (1). There are several types of ovarian carcinoma, but epithelial ovarian carcinoma (EOC) is the most frequent one, representing $>90 \%$ of all ovarian cancers (2). Historically, treatment of ovarian cancer has involved surgery combined with platinum-based chemotherapy (3). The main factors affecting the prognosis of patients with ovarian cancer are advanced stage at diagnosis and primary or secondary chemotherapy drug resistance, especially for those persistent or recurrent ovarian carcinoma. The five-year survival rate for ovarian cancer patients is currently $30 \%$ (4). The molecular alterations of cisplatin (DDP)-resistant cancer cells have previously been researched, however, the underlying mechanisms promoting DDP resistance in ovarian cancer cells remain to be elucidated (5). Therefore, research on the mechanisms of DDP resistance and reversing drug resistance in EOC are particularly urgent for the patients with current and refractory EOC.

The endoplasmic reticulum (ER) is an essential cellular compartment for protein synthesis and maturation. It also has other functions, including calcium storage and maintenance in $\mathrm{Ca}^{2+}$ homeostasis, steroid synthesis, and lipid and glycogen synthesis (6). Various physiological and pathological conditions may affect ER homeostasis, ultimately causing ER stress (ERS) (4-9). Accumulation of misfolded proteins and alterations in $\mathrm{Ca}^{2+}$ homeostasis in ER results in ERS and activation of autophagy in carcinoma cells. Typically, autophagy activates pro-survival mechanisms, as well as cell death programs, particularly if autophagy activated following ERS is a pro-survival response to restore the ER homeostasis by clearing the unfolded aggregates (10). Several studies have reported that ERS induces autophagy in mammalian cancer cell lines and mouse embryonic fibroblasts $(11,12)$. Heat shock proteins function as molecular chaperones that regulate cellular homeostasis and promote cell survival responses. Inhibition of autophagy and molecular chaperones may be a suitable pharmacological target to promote apoptosis in tumor cells. A previous study revealed that Hsp27 was involved in DDPand ERS induced autophagy activation in HCC cells (13). It has also been suggested that autophagy may be more active in DDP-resistant ovarian cancer cells (14). The 78-kDa 
glucose-regulated protein 78 (GRP78), also known as BiP or HSPA5, is predominantly localized in the ER as a molecular chaperone, and is associated with regulating the ERS pathway. Induction of GRP78 has been recognized as a marker for ERS and the onset of the unfolded protein response (15). Previous studies have indicated that the mechanistic target of rapamycin (mTOR) and Beclin 1 may have important roles in regulating cell autophagy (16). The present study aimed to determine the ERS effect on the DDP sensitivity of SKOV3 ovarian cancer cells. On this basis, the mechanism of the drug resistance of ovarian cancer cells to DDP was studied, which may contribute to the reversal of DDP resistance in ovarian cancer cells.

\section{Materials and methods}

Cell lines and culture conditions. SKOV3 human ovarian cancer cells were obtained from the Chinese Academy of Medical Sciences (Beijing, China) and Peking Union Medical College (Beijing, China). Cell lines were cultured at $37^{\circ} \mathrm{C}$ in RPMI-1640 culture medium (Gibco; Thermo Fisher Scientific, Inc., Waltham, MA, USA) supplemented with $10 \%$ fetal bovine serum (Invitrogen; Thermo Fisher Scientific, Inc.) in an atmosphere containing $5 \% \mathrm{CO}_{2}$ and $90 \%$ air.

Reagents. Human immunodeficiency virus (HIV) proteinase inhibitor, Saquinavir, was purchased from R\&D Systems, Inc., (Minneapolis, MN, USA). 3-Methyladenine (3-MA), an autophagy inhibitor, was purchased from Sigma-Aldrich (Merck Millipore, Darmstadt, Germany). Protein kinase inhibitor, LY294002, was obtained from Promega Corp. (Madison, WI, USA). GRP78, mTOR, Beclin 1 and $\beta$-actin antibodies were obtained from Santa Cruz Biotechnology, Inc., (Dallas, TX, USA). First Strand cDNA Synthesis kit was purchased from Beijing ComWin Biotech Co., Ltd (Beijing, China). BCA Protein Assay kit was purchased from Beyotime Institute of Biotechnology (Wuhan, China). Primers for mTOR, Beclin 1 and $\beta$-actin were acquired from Sangon Biotech Co., Ltd., (Shanghai, China)

Reverse transcription-quantitative polymerase chain reaction (RT-qPCR) analysis. Total RNA was extracted using TRIzol reagent (Invitrogen; Thermo Fisher Scientific, Inc.) and complementary DNA (cDNA) was synthesized using a First Strand cDNA Synthesis kit according to the manufacturer's instructions. Subsequently, $1 \mu 1$ synthesized cDNA was used for each PCR with each primer pair. qPCR was performed with 2X EasyTaq ${ }^{\circledR}$ PCR SuperMix kit (Beijing Transgen Biotech Co., Ltd., Beijing, China), and $25 \mu \mathrm{l}$ of the reaction mixture was used in a qPCR program. The $25-\mu 1$ reaction mixture contained $1 \mu 1$ each primer, $12.5 \mu 1$ 2X PCR Mix, $1 \mu \mathrm{l}$ template cDNA and $\leq 9.5 \mu 1$ double-distilled water (catalog no. KGDN4500; Nanjing KeyGen Biotech Co., Ltd., Nanjing, China) The primers were synthesized by Sangon Biotech Co., Ltd., as follows: mTOR, forward 5'-TTGAGGTTGCTATGA CCAGAGAGAA-3' and reverse 5'-TTACCAGAAAGGACA CCAGCCAATG-3', 566 bp; Beclin 1, forward 5'-CAGTTT GGCACAATCAATAAC-3' and reverse 5'-CATCCATCC TGTAGGGAAGAC-3', 352 bp; $\beta$-actin, forward 5'-TGTTTG AGACCTTCAACACCC-3' and reverse 5'-AGCACTGTG TTGGCGTACAG-3', 340 bp. Protocol parameters were as follows: Initial incubation at $95^{\circ} \mathrm{C}$ for $10 \mathrm{~min}$, followed by 30 cycles of denaturation at $95^{\circ} \mathrm{C}$ for $30 \mathrm{sec}$, annealing $\left(55^{\circ} \mathrm{C}\right)$ for $30 \mathrm{sec}$, elongation at $72^{\circ} \mathrm{C}$ for $1 \mathrm{~min}$ and a final extension at $72^{\circ} \mathrm{C}$ for $8 \mathrm{~min}$. The experiment was repeated three times, and the efficiency of cDNA synthesis from each sample was estimated using $\beta$-actin primers, which served as an endogenous control. Relative gene expression levels were calculated and analyzed using Quantity One analysis software (Bio-Rad Laboratories, Inc., Hercules, CA, USA). Data were analyzed using the $2^{-\triangle \Delta \mathrm{Cq}}$ method (17).

Western blot analysis. Cells were harvested and lysed in radioimmunoprecipitation assay buffer. Protein concentration was determined using a BCA Protein Assay kit. Denatured proteins $(50 \mu \mathrm{g})$ were separated by $12 \%$ SDS-PAGE and transferred to polyvinylidene difluoride membranes using the Bio-Rad electro-transfer system (Bio-Rad Laboratories, Inc.). The membranes were incubated with antibodies to visualize the proteins. Following blocking with $5 \%$ w/v non-fat dried milk for $1 \mathrm{~h}$, the membranes were incubated overnight with primary antibodies against GRP78 (1:500; bs-1219R), mTOR (1:500; bs-1992R) and Beclin 1 (1:500; bs-1353R) (all from Beijing Biosynthesis Biotechnology Co., Ltd., Beijing, China), rinsed with TBS-Tween 20 , and subsequently incubated at $37^{\circ} \mathrm{C}$ for $2 \mathrm{~h}$ with anti-rabbit immunoglobulin $\mathrm{G}$ secondary antibody conjugated to horseradish peroxidase (sc-2004; Santa Cruz Biotechnology, Inc.) at a dilution of 1:1,000. After applying Enhanced Chemiluminescent Plus detection reagents (EMD Millipore, Billerica, MA, USA), protein bands were visualized using an X-ray film (Fujifilm, Tokyo, Japan). $\beta$-actin was used as an internal control for protein loading and analysis. Quantitation of band intensity was performed by densitometry using Quantity One 1-D analysis software version 4.6.9 (Bio-Rad Laboratories, Inc.). The experiment was conducted three times.

MTT assay. MTT assays were used to assess the number of viable cells. After cells $\left(180-\mu 1\right.$ solution containing $1.0 \times 10^{5}$ cells $\left./ \mathrm{ml}\right)$ were cultured for $24 \mathrm{~h}$ in 96 -well plates $\left(1.8 \times 10^{4}\right.$ cells/well), culture medium was removed and replaced by $200 \mu$ l complete culture medium containing DDP. Final concentrations of DDP were $100,50,25,12.5,6.25,3.125,1.56$ and $0.78 \mu \mathrm{g} / \mathrm{ml}$, respectively. Each treatment was repeated in four wells. The control group received the same volume of culture medium with cells, without drugs, whereas blank wells contained the same volume of culture medium without cells and drugs. Cells were cultured for $72 \mathrm{~h}$. Cell viability was assessed using the MTT colorimetric assay. Briefly, $20 \mu 1$ MTT was added and incubated for $4 \mathrm{~h}$. Subsequently, $150 \mu \mathrm{l}$ dimethyl sulfoxide was added to dissolve the formazan crystals. Following shaking for $10 \mathrm{~min}$, the absorbance values were measured at a wavelength of $570 \mathrm{~nm}$ using a microplate reader (Molecular Devices, Sunnyvale, CA, USA). The experiment was repeated three times. The DDP half maximal inhibitory concentration $\left(\mathrm{IC}_{50}\right)$ in SKOV3 cells was calculated.

Statistical analysis. All values were shown as the mean \pm standard deviation from at least three independent experiments. Data were analyzed by Student's t-test (for comparing two intergroup results) or one-way analysis of variance with SPSS 17.0 (SPSS, Inc., Chicago, IL, USA). IC $_{50}$ was analyzed 
with the linear regression. $\mathrm{P}<0.05$ was considered to indicate a statistically significant difference.

\section{Results}

Expression of GRP78 in SKOV3 tumor cells. As an ER chaperone, GRP78 is commonly used as a marker of ERS. Western blotting analysis demonstrated that GRP78 protein expression levels were increased when tumor cells SKOV3 were treated with Saquinavir for $24 \mathrm{~h}$. Moreover, as shown in Table I and Fig. 1, the expression levels of GRP78 significantly increased following treatment with Saquinavir in a dose-dependent manner $(\mathrm{P}<0.05)$. These results were significantly different when compared with each other $(\mathrm{P}<0.05)$. These findings indicated that Saquinavir may lead to ERS, and that the level of ERS may be associated with the concentration of Saquinavir.

Effect of Saquinavir-induced ERS on the sensitivity of ovarian cancer cells to DDP. To confirm the effect of ERS on the sensitivity of SKOV3 ovarian cancer cells to DDP, the cells were treated with different concentrations of Saquinavir for $24 \mathrm{~h}$. The $\mathrm{IC}_{50}$ of the SKOV3 cells was $5.490 \pm 1.148 \mu \mathrm{g} / \mathrm{l}$. Following exposure to 10 and $20 \mu \mathrm{mol} / 1$ Saquinavir, the $\mathrm{IC}_{50}$ of SKOV3 cells significantly increased to $11.199 \pm 0.984$ and $14.906 \pm 2.015 \mu \mathrm{g} / \mathrm{l}$, respectively $(\mathrm{P}=0.003$ and $\mathrm{P}<0.001)$; however, no statistically significant difference was detected between the cells treated with 10 and $20 \mu \mathrm{mol} / 1$ Saquinavir $(\mathrm{P}=0.21$; Fig. 2). These findings suggest that Saquinavir resulted in ERS in SKOV3 tumor cells, which reduced the sensitivity of these ovarian cancer cells to DDP.

Effect of Saquinavir on protein and messenger RNA (mRNA) expression levels of mTOR and Beclin 1 in SKOV3 cells. Saquinavir, LY294002 (PI3K inhibitor) and DDP were added to the different cell groups, respectively, to final concentrations of 20, 20 and $5 \mu \mathrm{g} / \mathrm{ml}$, respectively. The expression levels of autophagy-related genes mTOR and Beclin 1 were assessed in SKOV3 ovarian cancer cells by RT-qPCR and western blotting. As shown in Tables II and III and Fig. 3, when the cells were exposed to Saquinavir for $24 \mathrm{~h}$, the protein and mRNA expression levels of mTOR and Beclin 1 in SKOV3 cells were increased. The results demonstrated that the protein and mRNA expression levels of mTOR and Beclin 1 in the Saquinavir + DDP group were significantly increased in comparison with the other groups. The Saquinavir group exhibited the next highest expression levels. Moreover, the protein and mRNA expression levels of mTOR and Beclin 1 in SKOV3 cells treated with Saquinavir were higher than those exhibited by cells in the LY294002 group. These results suggested that the PI3K inhibitor, LY294002, did not inhibit the mTOR expression completely through the PI3K/AKT/mTOR pathway. The protein and mRNA expression levels of mTOR and Beclin 1 among the groups of experiment were demonstrated to be significantly different $(\mathrm{F}=23.140, \mathrm{P}<0.001$ and $\mathrm{F}=24.389$, $\mathrm{P}<0.001$, respectively). Protein expression levels of mTOR and Beclin 1 among the groups were also significantly different $(\mathrm{F}=39.345, \mathrm{P}<0.001$ and $\mathrm{F}=261.877, \mathrm{P}<0.001$, respectively). However, further comparison indicated that the protein and mRNA expression levels of mTOR and Beclin 1 were significantly different between any two groups (control vs. DDP; control vs. Saquinavir + DDP; control vs. LY294002; control vs. Saquinavir; DDP vs. Saquinavir + DDP; DDP vs. Saquinavir; Saquinavir + DDP vs. LY294002; Saquinavir + DDP vs. Saquinavir; and LY294002 vs. Saquinavir), with the exception of the LY294002 and DDP groups.

Effect of autophagy suppression on the sensitivity of ovarian cancer cells to DDP. The present study confirmed that Saquinavir resulted in ERS in SKOV3 cells, which reduced the sensitivity of ovarian cancer cells to DDP. Furthermore, it demonstrated that the sensitivity of ovarian cancer cells to DDP was associated with the level of ERS. In the present study, SKOV3 cells were exposed to the autophagy inhibitor, 3-MA, and various concentrations of Saquinavir. $\mathrm{IC}_{50}$ values in the control, Saquinavir $10 \mu \mathrm{mol} / 1$, Saquinavir $10 \mu \mathrm{mol} / 1+3-\mathrm{MA}$, Saquinavir $20 \mu \mathrm{mol} / 1$ and Saquinavir $20 \mu \mathrm{mol} / 1+3$-MA groups were $5.490 \pm 1.148,11.199 \pm 0.984$, $6.624 \pm 0.218,14.906 \pm 2.015$ and $7.888 \pm 0.086 \mu \mathrm{g} / \mathrm{ml}$, respectively (Fig. 4). IC $_{50}$ of DDP was decreased in SKOV3 cells after 3-MA combined with Saquinavir treatment compared with Saquinavir treatment alone. This indicated that the autophagic response induced by Saquinavir treatment was inhibited by 3-MA. These results suggest that the sensitivity to DDP was significantly improved in SKOV3 cells after 3-MA exposure $(\mathrm{F}=31.898, \mathrm{P}<0.001)$.

\section{Discussion}

ER is an essential intracellular organelle with multiple roles including the synthesis, folding, assembly and maturation of nascent proteins, $\mathrm{Ca}^{2+}$ storage, glycosylation, and the trafficking of newly-synthesized membrane and secretory proteins (18). Perturbations of these processes have been demonstrated to interfere with the proper functioning of ER, thus leading to a condition defined as ERS $(8,9)$. Previous studies have shown that DDP resistance in cancer cells was associated with ERS $(19,20)$. DDP results in the accumulation of unfolded and misfolded proteins in the lumen of the ER. This ERS subsequently induces autophagy. The majority of accumulated proteins and soluble proteins were cleared by autophagy pathways and tumor cells are able to survive $(21,22)$. It has been demonstrated that tunicamycin, an ERS inducer, augmented DDP cytotoxicity by upregulating ERS-mediated apoptosis, indicating that autophagy has an important role in preventing DDP-induced apoptosis in HeLa cells (23). Saquinavir, which is a HIV proteinase inhibitor, is able to induce ERS in various tumors, including NC160 cells (24), non-small cell lung cancer (25) and melanocytoma (26). McLean et al (27) have previously shown that Saquinavir leads to ERS and cell autophagy.

Altering the tumor microenvironment and growth patterns could activate ERS. The survival of cells upon undergoing ERS induces better adaptation to various physiological and pathological conditions, which is one of the important mechanisms in tumor cells remaining malignant and promoting drug resistance (28). GRP78 has been well-established as an ER chaperone and is widely used as a marker for ERS (29). Our study confirmed that Saquinavir leads to ERS in SKOV3 tumor cells, which reduces the sensitivity of ovarian cancer cells to 
Table I. Relative protein expression levels of GRP78 following treatment with Saquinavir.

\begin{tabular}{lc}
\hline Groups & GRP78 \\
\hline Control & $0.361 \pm 0.009$ \\
Saquinavir $10 \mu \mathrm{mol} / 1$ & $0.446 \pm 0.010$ \\
Saquinavir $20 \mu \mathrm{mol} / 1$ & $0.489 \pm 0.105$ \\
Saquinavir $40 \mu \mathrm{mol} / 1$ & $0.511 \pm 0.010$ \\
\hline
\end{tabular}

Data are presented as the mean \pm standard deviation. GRP78, glucose-related protein 78 .



Figure 1. GRP78 expression levels in SKOV3 ovarian cancer tumor cells. (A) GRP78 protein expression levels following treatment with 0,10,20 and $40 \mu \mathrm{mol} / 1$ Saquinavir in SKOV3 cells, as determined by western blotting. (B) Subsequent quantitative analysis of GRP78 protein expression levels. GRP78, glucose-related protein 78.

DDP. Furthermore, the sensitivity of ovarian cancer cells to DDP appears to be associated with the level of ERS. 3-MA is a specific inhibitor of the autophagic pathway. After SKOV3 tumor cells are exposed to the autophagy inhibitor 3-MA, sensitivity of ovarian cancer cells to DDP could be effectively reversed. Therefore, it was speculated that ERS may induce DDP resistance through enhanced autophagy in SKOV3 cells. This demonstrated that an increase in the level of ERS had an important role in DDP resistance in ovarian cancer, particularly secondary DDP resistance associated with continuous ERS and an increase in the level of autophagy in ovarian cancer exposed to DDP, a chemotherapeutic agent, periodically.

Accumulating evidence has indicated that ERS is associated with tumor cell survival, tumor progression and chemotherapy resistance (29-31); however, its precise mechanism remains unclear. Previous studies have shown that the PI3K/Akt $/ \mathrm{mTOR}$ pathway is involved in ERS-triggered apoptosis, and is also associated with the regulation of autophagy $(32,33)$; however, PI3K inhibitor did not inhibit the expression of mTOR completely. This suggested other signaling pathways may exist, requiring further investigation (34).
Table II. Expression of mTOR and Beclin 1 messenger RNA in SKOV3 human ovarian cancer cells.

\begin{tabular}{lcc}
\hline Groups & mTOR & Beclin 1 \\
\hline Control & $0.287 \pm 0.073$ & $0.342 \pm 0.029$ \\
DDP & $0.468 \pm 0.031$ & $0.456 \pm 0.028$ \\
Saquinavir + DDP & $0.684 \pm 0.072$ & $0.647 \pm 0.047$ \\
LY294002 & $0.467 \pm 0.005$ & $0.477 \pm 0.056$ \\
Saquinavir & $0.5772 \pm 0.016$ & $0.565 \pm 0.037$ \\
\hline
\end{tabular}

Data are presented as the mean \pm standard deviation. DPP, cisplatin; mTOR, mechanistic target of rapamycin.

Table III. Expression of mTOR and Beclin 1 proteins in SKOV3 human ovarian cancer cells.

\begin{tabular}{lcc}
\hline Groups & mTOR & Beclin 1 \\
\hline Control & $0.218 \pm 0.038$ & $0.265 \pm 0.033$ \\
DDP & $0.450 \pm 0.045$ & $0.345 \pm 0.029$ \\
Saquinavir + DDP & $0.624 \pm 0.058$ & $0.924 \pm 0.033$ \\
LY294002 & $0.453 \pm 0.041$ & $0.384 \pm 0.030$ \\
Saquinavir & $0.544 \pm 0.019$ & $0.712 \pm 0.024$
\end{tabular}

Data are presented as the mean \pm standard deviation. DPP, cisplatin; mTOR, mechanistic target of rapamycin.



Figure 2. Sensitivity of SKOV3 human ovarian cancer cells to cisplatin decreased after treatment with 10 and $20 \mu \mathrm{mol} / 1$ Saquinavir. $\mathrm{IC}_{50}$, half maximal inhibitory concentration.

Autophagy is a 'self-eating' process by which a cell digests damaged organelles or misfolded proteins by sequestering the target cargo in a double membrane and fusing to lysosomes for degradation, thereby supplementing intermediate metabolism with the products of digestion $(35,36)$. mTOR has a critical role in the initiation of the autophagic process. mTOR activation is able to inhibit autophagy. Beclin 1, which is a mammalian autophagy gene, was the first protein that was demonstrated 
A

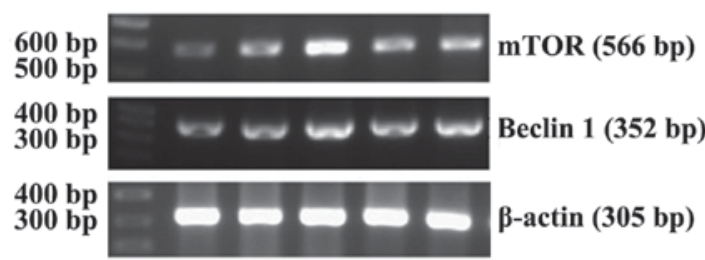

C

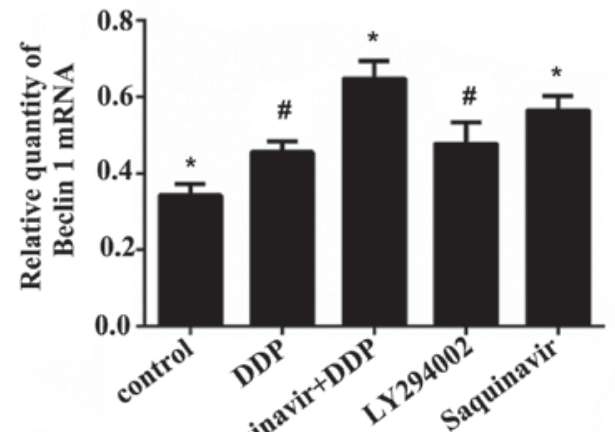

E

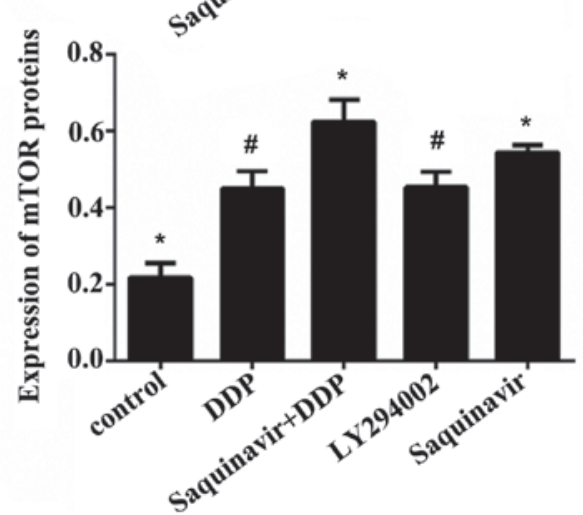

B
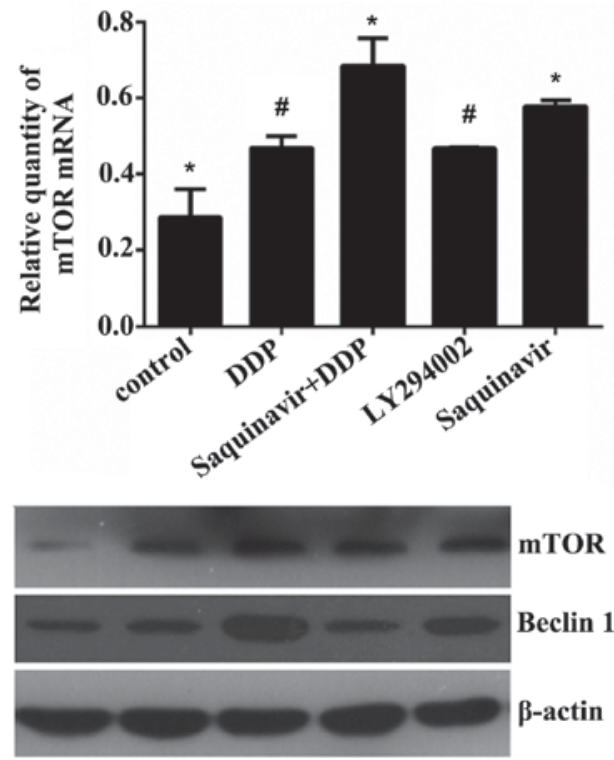

F

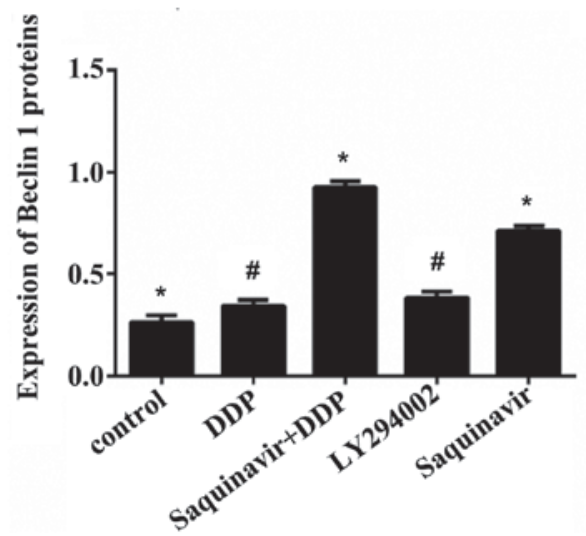

Figure 3. Effect of Saquinavir on the (A-C) mRNA and (D-F) protein expression levels of mTOR and Beclin 1 in SKOV3 cells. (A) mRNA expression of mTOR and Beclin 1 in SKOV3 cells. (B) Bar chart of mTOR mRNA expression in SKOV3 cells. (C) Bar chart of Beclin 1 mRNA expression in SKOV3 cells. (D) Protein expression levels of mTOR and Beclin 1 in SKOV3 cells. (E) Bar chart of mTOR protein expression of in SKOV3 cells. (F) Bar chart of Beclin 1 protein expression in SKOV3 cells. Data are presented as the mean + standard deviation. ${ }^{*} \mathrm{P}<0.001$ vs. all other groups. ${ }^{~} \mathrm{P}<0.001$ vs.control, Saquinavir $+\mathrm{DDP}$ and Saquinavir groups. mTOR, mechanistic target of rapamycin; DDP, cisplatin; mRNA, messenger RNA.

to induce autophagy (37). Studies have identified that cell autophagy has a critical role in the occurrence and development of tumor cells, and it has been suggested that autophagy may have a role in cancer cell chemoresistance $(38,39)$. A previous study researched co-treatment of DDP with trifluoperazine, an inducer of autophagy, which sensitized H460/cis DDP-resistant lung carcinoma cells to DDP, suggesting that the decreased levels of autophagy may promote DDP resistance in lung cancer (40). Another previous study suggested that ERS induced apoptosis and led to DDP resistance in human ovarian cancer cells, but provided limited information on the role of autophagy in DDP resistance (41). ERS may regulate autophagy through mTOR and Beclin 1 pathways. The present study further explored that when ERS was induced by Saquinavir in tumor cells SKOV3, the expression levels of mTOR and Beclin 1 were upregulated, decreasing the sensitivity of ovarian cancer cells to DDP. We hypothesize that ovarian cancer cells experienced chemotherapy or radiotherapy, and numerous organelles or proteins were destroyed in ovarian cancer cells. Enhanced cell autophagy would aid the clearance of these harmful substances to maintain stabilization of homoeostasis and the cells could prevent from death. Cells benefit from moderate ERS to alleviate damage,

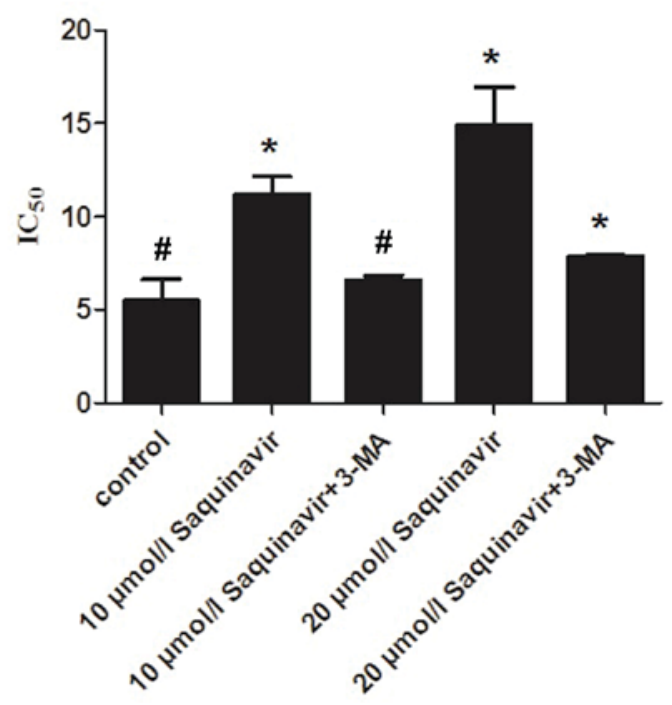

Figure 4. Effect of autophagy suppression on the sensitivity of SKOV3 ovarian cancer cells to cisplatin. ${ }^{*} \mathrm{P}<0.001$ vs. all other groups. ${ }^{\#} \mathrm{P}<0.001$ vs. $10 \mu \mathrm{mol} / 1$ Saquinavir, $20 \mu \mathrm{mol} / 1$ Saquinavir and $20 \mu \mathrm{mol} / 1$ Saquinavir + 3-MA. 3-MA, 3-methyladenine; $\mathrm{IC}_{50}$, half maximal inhibitory concentration. 
whereas sustained ERS induces cell death (42). Continuous autophagy in cells leads to the breakdown of important organelles and proteins and eventual cell death via autophagy. To prevent this type of cell death, the PI3K/AKT/mTOR pathway is activated in ovarian cancer cells to appropriately suppress autophagy. In the present study, it was also demonstrated that the expression levels of mTOR were upreguated, which suggested autophagy was inhibited. Our findings have identified that downregulating autophagy via 3-MA may prevent the effect of Saquinavir-induced ERS on the sensitivity of ovarian cancer cells to DDP. Therefore, we hypothesize that an increase in the level of autophagy had a dominant role in the ERS activation of ovarian cancer cells and decreased the drug sensitivity of ovarian cancer to DDP.

In conclusion, mTOR and Beclin 1 may be important in the regulation of cell autophagy. ERS acts like a double-edged sword, since it can induce apoptosis and promote cell survival. The present findings demonstrated that ERS was able to promote cell survival through regulation of the level of autophagy and have a role in protecting cell from being destroyed. It was speculated that ERS and enhanced cell autophagy were important mechanisms which resulted in DDP resistance in ovarian cancer. Targeting ERS or inhibiting autophagy may be an encouraging technique to overcome chemotherapeutic resistance.

\section{Acknowledgements}

The authors would like to thank Tianjin Medical University (Heping, China) and all the teachers in our lab, particularly Professor Hao Zhang and Professor Zheng Su, for their assistance during the experiment.

\section{References}

1. Khanra K, Panda K, Mitra AK, Sarkar R, Bhattacharya C and Bhattacharya N: Exon 8-9 mutations of DNA polymerase $\beta$ in ovarian carcinoma patients from Haldia, India. Asian Pac J Cancer Prev 13: 4183-4186, 2012.

2. Ho CM, Chanq SF, Hsiao CC, Chien TY and Shih DT: Isolation and characterization of stromal progenitor cells from ascites of patients with epithelial ovarian adenocarcinoma. J Biomed Sci 19: 23, 2012.

3. Romanidis K, Nagorni EA, Halkia E and Pitiakoudis M: The role of cytoreductive surgery in advanced ovarian cancer: The general surgeon's perspective. J BUON 19: 598-604, 2014.

4. Siddik ZH: Cisplatin: Mode of cytotoxic action and molecular basis of resistance. Oncogene 22: 7265-7279, 2003.

5. Galluzzi L, Senovilla L, Vitale I, Michels J, Martins I, Kepp O, Castedo M and Kroemer G: Molecular mechanisms of cisplatin resistance. Oncogene 31: 1869-1883, 2012.

6. Pang XL, He G, Liu YB, Wang Y and Zhang B: Endoplasmic reticulum stress sensitizes human esophageal cancer cell to radiation. World J Gastroenterol 19: 1736-1748, 2013.

7. Haeri $M$ and Knox BE: Endoplasmic reticulum stress and unfolded protein response pathways: Potential for treating age-related retinal degeneration. J Ophthalmic Vis Res 7: 45-59, 2012.

8. Walter P and Ron D: The unfolded protein response: From stress pathway to homeostatic regulation. Science 334: 1081-1086, 2011

9. Tabas I and Ron D: Integrating the mechanisms of apoptosis induced by endoplasmic reticulum stress. Nat Cell Biol 13: 184-190, 2011.

10. Verfaillie T, Salazar M, Velasco G and Agostinis P: Linking ER stress to autophagy: Potential implications for cancer therapy. Int J Cell Biol 2010: 930509, 2010.

11. Kang JH, Chang YC and Maurizi MR: 4-O-carboxymethyl ascochlorin causes ER stress and induced autophagy in human hepatocellular carcinoma cells. J Biol Chem 287: 15661-15671, 2012.
12. Liu D, Yang Y, Liu Q and Wang J: Inhibition of autophagy by 3-MA potentiates cisplatin-induced apoptosis in esophageal squamous cell carcinoma cells. Med Oncol 28: 105-111, 2011.

13. Chen R, Dai RY, Duan CY, Liu YP, Chen SK, Yan DM, Chen $\mathrm{CN}$, Wei $\mathrm{M}$ and $\mathrm{Li} \mathrm{H}$ : Unfolded protein response suppresses cisplatin-induced apoptosis via autophagy regulation in human hepatocellular carcinoma cells. Folia Biol (Praha) 57: 87-95, 2011.

14. Bao LJ, Jaramillo MC, Zhang Z, Zheng Y, Yao M, Zhang DD and Yi X: Induction of autophagy contributes to cisplatin resistance in human ovarian cancer cells. Mol Med Rep 11: 91-98, 2015.

15. Lee AS: GRP78 induction in cancer: Therapeutic and prognostic implications. Cancer Res 67: 3496-3499, 2007.

16. Kang R, Zeh HJ, Lotze MT and Tang D: The Beclin 1 network regulates autophagy and apoptosis. Cell Death Differ 18: 571-580, 2011.

17. Livak KJ and Schmittqen TD: Analysis of relative gene expression data using real-time quantitative PCR and the 2(-Delta Delta C(T)) method. Method 4: 402-408, 2001.

18. Kato $\mathrm{H}$ and Nishitoh H: Sress responses from the endoplasmic reticulum in cancer. Front Oncol 5: 93, 2015.

19. Shi S, Xu H, Li C, et al: Endoplasmic reticulum stress and tumor. Medical Recapitulate 15: 525-527, 2009.

20. Yuan YF: Endoplasmic reticulum stress and apoptosis of tumor cells. J Mol Diagn Ther 2: 128-134, 2010.

21. Liu Y and Ye Y: Roles of p97-associated deubiquitinases in protein quality control at the endoplasmic reticulum. Curr Protein Pept Sci 13: 436-446, 2012.

22. White MC, Johnson GG, Zhang W, Hobrath JV, Piazza GA and Grimaldi M: Sulindac sulfide inhibits sarcoendoplasmic reticulum $\mathrm{Ca} 2+$ ATPase, induces endoplasmic reticulum stress response and exerts toxicity in glioma cells: Relevant similarities to and important differences from celecoxib. J Neurosci Res 91: 393-406, 2013.

23. Xu Y, Yu H, Qin H, Kang J, Yu C, Zhong J, Su J, Li H and Sun L: Inhibition of autophagy enhances cisplatin cytotoxicity through endoplasmic reticulum stress in human cervical cancer cells. Cancer Lett 314: 232-243, 2012.

24. Gills JJ, Lopiccolo J, Tsurutani J, Shoemaker RH, Best CJ, Abu-Asab MS, Borojerdi J, Warfel NA, Gardner ER, Danish M, et al: Nelfinavir, a lead HIV protease inhibitor, is a broad-spectrum, anticancer agent that induces endoplasmic reticulum stress, autophagy, and apoptosis in vitro and in vivo. Clin Cancer Res 13: 5183-5194, 2007.

25. Gills JJ, Lopiccolo J, Tsurutani J, Shoemaker RH, Best CJ, Abu-Asab MS, Borojerdi J, Warfel NA, Gardner ER, Danish M, et al: Nelfinavir, a lead HIV protease inhibitor, is a broad-spectrum, anticancer agent that induces endoplasmic reticulum stress, autophagy, and apoptosis in vitro and in vivo. Clin Cancer Res 13: 5183-5194, 2007.

26. Jiang W, Mikochik PJ, Ra JH, Lei H, Flaherty KT, Winkler JD and Spitz FR: HIV protease inhibitor nelfinavir inhibits growth of human melanoma cells by induction of cell cycle arrest. Cancer Res 67: 1221-1227, 2007.

27. McLean K, VanDeVen NA, Sorenson DR, Daudi S and Liu JR: The HIV protease inhibitor saquinavir induces endoplasmic reticulum stress, autophagy, and apoptosis in ovarian cancer cells. Gynecol Oncol 112: 623-630, 2009.

28. Feldman DE, Chauhan V and Koong Ac: The unfolded protein response: A novel component of the hypoxic stress response in tumors. Mol Cancer Res 3: 597-605, 2005.

29. Ni M and Lee AS: ER chaperones in mammalian development and human diseases. FEBS Lett 581: 3641-3651, 2007.

30. Lin Y, Wang Z, Liu L and Chen L: Akt is the downstream target of GRP78 in mediating cisplatin resistance in ER stress-tolerant human lung cancer cells. Lung Cancer 71: 291-297, 2011.

31. Jiang CC, Mao ZG, Avery-Kiejda KA, Wade M, Hersey P and Zhang XD: Glucose regulatedprotein 78 antagonizes cisplatin and adriamycin in human melanoma cells. Carcinogenesis 30: 197-204, 2009.

32. Kato H, Nakajima S, Saito Y, Takahashi S, Katoh R and Kitamura M: mTORC1 serves ER stress-triggered apoptosis via selective activation of the IRE1-JNK pathway. Cell Death Differ 19: 310-320, 2012.

33. Oh SH and Lim SC: Endoplasmic reticulum stressmediated autophagy/apoptosis induced by capsaicin (8-methyl-N-vanillyl-6-nonenamide) and dihydrocapsaicin is regulated by the extent of c-Jun NH2-terminal kinase/extracellular signal-regulated kinase activation in WI38 lung epithelial fibroblast cells. J Pharmacol Exp Ther 329: 112-122, 2009. 
34. Kadowaki H, Nishitoh $\mathrm{H}$ and Ichijo H: Survival and apoptosis signals in ER stress: The role of protein kinases. J Chen Neuroanat 28: 93-100, 2004.

35. Clarke R, Cook KL, Hu R, Facey CO, Tavassoly I, Schwartz JL, Baumann WT, Tyson JJ, Xuan J, Wang Y, et al: Endoplasmic reticulum stress, the unfolded protein response, autophagy, and the integrated regulation of breast cancer cell fate. Cancer Res 72: 1321-1331, 2012.

36. He C and Klionsky DJ: Regulation mechanisms and signaling pathways of autophagy. Ann Rev Genet 43: 67-93, 2009

37. Takahashi Y, Coppola D, Matsushita N, Cualing HD, Sun M Sato Y, Liang C, Jung JU, Cheng JQ, Mulé JJ, et al: Bif-1 interacts with Beclin1 through UVRAG and regulates autophagy and tumorigenesis. Nat Cell Biol 9: 1142-1151, 2007.
38. Carew JS, Nawrocki ST and Cleveland JL: Modulating autophagy for therapeutic benefit. Autophagy 3: 464-467, 2007.

39. Mathew R, Karantza-Wadsworth V and White E: Role of autophagy in cancer. Nat Rev Cancer 7: 961-967, 2007.

40. Sirichanchuen B, Pengsuparp T and Chanvorachote P: Long-term cisplatin exposure impairs autophagy and causes cisplatin resistance in human lung cancer cells. Mol Cell Biochem 364: 11-18, 2012.

41. Yu H, Su J, Xu Y, Kang J, Li H, Zhang L, Yi H, Xiang X, Liu F and Sun L: p62/SQSTM1 involved in cisplatin resistance in human ovarian cancer cells by clearing ubiquitinated proteins. Eur J Cancer 47: 1585-1594, 2011.

42. Schönthal AH: Pharmacological targeting of endoplasmic reticulum stress signaling in cancer. Biochem Pharmacol 85: 653-666, 2013. 